\title{
Heuristic Techniques as Part of Heuristic Methods and Interaction of Personality Types in their Application
}

\author{
Viktor Ivanov*,1, Lubomir Dimitrov ${ }^{2}$, Svitlana Ivanova $^{3}$, Olena Olefir $^{4}$ \\ ${ }^{1}$ Odessa National Polytechnic University, Department Mechanical Engineering and Elements of Machine, Odesa, 65044, Ukraine \\ ${ }^{2}$ Technical University of Sofia, Department Mechanical Engineering, Sofia, 1000, Bulgaria
}

${ }^{3}$ Department Mathematics and its Teaching Methods, South Ukrainian National Pedagogical University named after K.D. Ushynsky, Odesa, 65020, Ukraine

${ }^{4}$ Department Advanced Mathematics and Statistics of Affiliation, South Ukrainian National Pedagogical University named after K.D. Ushynsky, Odesa, 65020, Ukraine

\begin{tabular}{l} 
A R T I C L E I N F O \\
\hline Article history: \\
Received: 08 November, 2020 \\
Accepted: 24 December, 2020 \\
Online: 15 January, 2021
\end{tabular}

Keywords:

Heuristic technique

Personality types

Generalized heuristic method

\begin{abstract}
A B S T R A C T
The widespread team project method is more effective when used in conjunction with heuristic methods. The large number of heuristic methods and the variety of their descriptions create a problem to prepare students for the use of these methods. A method based on two areas of knowledge - heuristics and psychology - is proposed. The personality types of students STEM specialties according to Myers-Briggs are considered. An analysis of interaction of personality types from the point of view of application of heuristic methods is performed. The survey for percentage composition personality types of student STEM specialties was carried out and predominantly types of student STEM specialties was determine. Heuristic methods are consideration as sum of heuristic techniques and procedure. It is shown that many methods involve the same heuristic techniques and differ only in procedures. A generalized method has been developed that allows replacing most of the methods based on collective discussion. This method included five heuristic techniques: collective discussion, pause between the presentation of ideas and their criticism, random associations, analogy, expert evaluation, using a matrix. This method is mainly aimed at teaching students of STEM specialties. A project team is formed to use the method. The composition of this team includes a discussion group, a criticism group and a expert evaluation group. These groups are formed in accordance with the personal types of participants. The method includes an algorithm for team members to interact when using heuristic techniques and procedures.
\end{abstract}

\section{Introduction}

Research in the field of heuristics is carried out within a many scientific disciplines. Different approaches and terminology are used. In philosophical works, heuristics is understood as a science, the subject of which is the process of solving a problem in conditions of uncertainty. Another aspect of heuristics is the branch of the science of thinking. Different interpretation is related to pedagogy, so heuristics are often understood as a way of learning. The concept of heuristic is also widely used in

*Corresponding Author: Viktor Ivanov, ivv@opu.ua

WWw.astesj.com

https://dx.doi.org/10.25046/aj060123 cybernetics, where it is interpreted as a heuristic algorithm. Researchers estimate the number of existing heuristic methods in different ways - from several to hundreds. There is no unambiguous idea about the structure of the heuristic method. In different scientific disciplines, a set of principles, a set of steps, or a set of heuristic techniques are considered to constitute a method. First of all, heuristic methods aimed at enhancing the creative abilities of students and involving them in collective creativity are relevant for teaching. The number of such methods is quite large, but there are many common features in the structure of these methods. When it comes to collective creativity, the question of 
performers immediately arises. How do personality differences between people affect their use of heuristic methods and how do these differences affect the organization of their interaction? This paper, that is an extension of work originally presented in International Conference on High Technology for Sustainable Development, is dedicated to solving this problem [1].

Consideration of the application of heuristic methods is associated with the personal differences of performers in two aspects: the effectiveness of the application of methods and teach students to use heuristic methods. Many heuristic methods are focused on collective creativity. Methods of this type are well suited for use in student learning. Heuristic methods are most widely used in teaching students studying the following specialties: science, technology, engineering and mathematics (STEM). When using heuristic methods formed by a group of students working together. The interaction of performers in such a group is well studied in project management. This kind of group of performers is called a project team. A large number of publications on psychology, management and education are devoted to organizing the work of the project team and the selection of performers. The project team can be organized for one seminar, or can work on a course project or diploma project during a semester or a year.

\section{Review of heuristic methods}

As methods of generating ideas used in teaching, the following are considered: Brainstorming, Method 6-3-5, C-Sketch, Gallery, Brainsketching. These methods differ in the form of communication: verbal - brainstorming; text - method 6-3-5; graphic - method C-Sketch; sketch, discussion and text - Gallery; sketch and text - Brainsketching [2]. In the 6-3-5 method, six participants write 3 ideas on a piece of paper in 5 minutes. Then they exchange their notes. This method resembles individual brainstorming in that each participant generates ideas individually, as well as electronic or online electronic brainstorming in which participants exchange ideas via e-mail. In Brainsketching, participants present their ideas in the form of sketches and then exchange them. As in the 6-3-5 method, the criticism of ideas is carried out without the participation of those who put forward the ideas. It is assumed that the graphical representation of ideas will help to find associations. In the Gallery method, the sketches created are discussed and selected together. In the C-Sketch method, the sketch is an imaginary construction. This method is targeted at mechanical engineers.

An experiment comparing Brainsketching and Brainstorming methods has been carried out. The experiment showed that during the Brainstorming process, the participants produced more ideas, and during Brainsketching, the ideas were more original. It is noted that Brainsketching is a type of Brainwriting. That is, the participants put forward ideas individually, as in the 6-3-5 method, only in the form of a sketch [3].

The methods can be divided into three groups [4]. Methods using graphic presentation of information: C-Sketch and Gallery. The methods of using the text include: Brainstorming, K-J Method, Checklists, Affinity Method, Storyboarding, Fishbone, and Method 6-3-5. In addition to the above two groups of methods, another group of Cross-representation methods is proposed [4].
In the Storyboarding method, the sketch is not static but is created in the presence of the discussion group members. The purpose of a sketch or series of sketches is to represent the origin and development of an idea. Affinity Methods operate with different types of information presentation: text, photo, sketch, etc. The group discussion task is sorting similar information into clusters. Affinity Method is, as many believe, a variation of the K$\mathrm{J}$ method. In the Fishbone method, as a result of collective discussion, the factors are also grouped into clusters; only the grouping is visualized as a single diagram.

Cross-representation methods include Morphological analysis and Synectics [4]. Synectics uses collective discussion, the prohibition of criticism as in Brainstorming. Synectics encourages the use of analogies and introspection of the idea generation process. Morphological analysis requires the identification of a set of functions and a possible set of design solutions or physical principles. For this, the analogy of physical processes is widely used [5]. The comparison of possible combinations of properties and design solutions is performed using a matrix. Another name for the method is morphological matrix [5]. Note that the same method has different names Morphological analysis and Morphological matrix. The Affinity method is also called the Affinity Diagram; some researchers describe it as a separate method, others as another name for the K-J method. Brainwriting is an individualized brainstorming session and is very similar to the 6-3-5 method. The peculiarity of publications on heuristics is the use of different names of methods and different points of view on their classification, which is partly due to the authors' affiliation to different scientific disciplines, as well as different ways of presenting information by representatives of the humanities and engineering disciplines.

The comparison of the methods Brainwriting and S.C.A.M.P.E.R. was carried out [6]. In S.C.A.M.P.E.R. method the ideas are generated based on responses to prompts: Substitute, Combine, Modify, etc. The study did not obtain data that could indicate the superiority of one of the methods. It is noted that more creativity can be achieved if the participants are from different universities and different countries. This can be interpreted as a recommendation for using electronic Brainstorming.

The comparison of methods S.C.A.M.P. and TRIZ are performed; also data about Brainstorming was used [7]. It is noted that Brainstorming allows for more creative ideas, but S.C.A.M.P.E.R. and TRIZ provided ideas that could be implemented. Method TRIZ allowed getting more creative ideas than the S.C.A.M.P.E.R method. The S.C.A.M.P.E.R method is presented as intuitive in contrast to the logical TRIZ method.

It should be noted that TRIZ is aimed at solving inventive tasks, that is, it is focused at engineers. More precisely, the name is TRIZ methodology, not a method, since TRIZ contains a set of methods. Most of the TRIZ methods are not methods, but are recommendations for a mechanical engineer, for example: replace sliding friction with rolling friction or replace rectilinear motion with rotational. At the same time provides a method of analogy and decomposition.

A comparison of the methods used in the educational process is carried out; these methods include: Brainstorming, Method 6-35, Morphological matrix, Mind and Brain Mapping, Patent 
Research, AIDA, Function Structure, House of Quality, Black Box, Decision, Matrix and Pugh Chart. The selection of methods raises questions - how and for what purposes these methods are used in the educational process [8]. The following design stages are highlighted: Task planning and clarification, Conceptual design, Embodiment design, Detailed design [9]. The advantages of the AIDA method are noted, which can be used both at the Task planning and clarification stage and at the Conceptual design stage. The possibility of using one AIDA method instead of using a number of methods is being considered: Brainstorming, Black Box, Morphological matrix, Decision Matrix and Pugh Chart [8]. Note that the feature of the AIDA method is the construction of an option graph, and then the established relationships are analyzed in the matrix. In this part, the method is similar to the Morphological matrix and Decision Matrix methods.

There are different ideas about the stages of the creative process and the stages of the engineering design process [10]. A large number of classifications have a lot in common. Most researchers indicate Analysis phase as the first stage or phase, and Generation phase as the second stage, which is also called Ideafinding, Generating ideas. Inspiration, Response generation. The third stage is the Evaluation phase, which is also called Looking Back, Solution-finding, Reinterpretation, Response validation. The fourth stage is Communication / implementation or Acceptance-finding, Developing an implementation plan [10].

Taking into account the division of the creative process into stages, it is possible to assess the applicability of various methods to these stages. Undoubtedly, of the above methods used in teaching for Generation phase, methods of collective creativity are suitable: Brainstorming, Method 6-3-5, S.C.A.M.P.E.R., Brainwriting, Brainsketching, C-Sketch и Gallery. The mind and brain map is suitable for use by a teacher. If it is used by students, it is transformed into methods with a graphical representation of the idea - Brainsketching, C-Sketch and Gallery.

The methods K-J Method, Checklists, Affinity Method, Storyboarding, Fishbone, AIDA are more suitable for the Evaluation phase, although they are also used in the second stage. The Morphological matrix and Decision Matrix methods are ideal for the third stage. In the fourth stage, Pugh Chart and Checklists can be used. Decision Matrix also has another name Decision support matrix (DSM) this method is aimed at solving engineering problems. The method DSM allows to establish the presence or absence of interactions between the nodes of the product. Based on the found interactions, elements are combined into a cluster [11]. Another method using a matrix is the Interaction Matrix method. This method also is aimed at identifying the interactions between structural elements and their systematization [12].

The TRIZ methodology contains a set of methods that are suitable for each stage of the design process. At the same time, the core of the method is also a matrix - a matrix of contradictions. The TRIZ method involves the formation of a matrix, in which the components and elements of the product are located horizontally, and functions or parameters - vertically. The contents of the matrix cells are a list of methods that can be used to resolve technical contradictions [13]. To this series of methods that use a matrix should be added - a Matrix Diagram (Quality Table). The matrix includes elements between which it is necessary to establish the interaction. The values in the cells of the matrix indicate the presence and strength of the interaction [14].

\section{Myers-Briggs personality types and creative process}

The effectiveness of the application of heuristic methods largely depends on the selection of participants and the organization of their work. The selection of participants is especially important when the project team is being formed for a long time. The interaction of participants in the project team is described by the team role methodology. A team role is a description of a behaviour pattern that defines how one team member interacts with other team members when working to accomplish assigned tasks. In project management, the most widespread is the classification of team roles in accordance with [15]. Along with the Belbin typology, there are other typologies of team roles, including MTR-I Team Dynamics [16] and Margerison-McKenna [17]. In psychology, the typology of K. Jung and his followers is generally accepted. Thus, A. Augustinavichute proposed her original modification of K. Jung's typology, which was called "socionics" [18]. The most widely known indicator of the Myers-Briggs types (MBTI) [19]. The MBTI divides individual differences into four opposite pairs, leading to sixteen possible personality types. The sixteen types are usually designated by a four-letter abbreviation, the initial letters of each of their four preference types. For example: INTJ: Introversion (I), Intuition (N), Thinking (T), Judgment (J) or ESFP: Extraversion (E), Sensation (S), Feeling (F), Perception (P) $[19,20]$. Likewise, from a combination of four letters, abbreviations of the remaining fourteen personality types are formed.

It is important to note that the role of an individual in the project team may not correspond to his innate individual differences as determined by the Myers-Briggs test. Work experience, acquired qualifications affect the role of the individual in the project team and the results of the MTR-i and Belbin tests. The personality type, in accordance with the Myers-Briggs typology, reflects the individual differences of a person, to a lesser extent dependent on external circumstances and experience, and to a greater extent - on the innate characteristics of the nervous system. Innate human abilities are important in terms of the spontaneous expression of ideas on which most heuristic methods are based. Therefore, this typology is most suitable for describing the heuristic abilities of project team members and their interaction in the collective generation of ideas, critical discussion and expert evaluation.

In the first place it is of interest the possible role of a certain type of students in the process of collective discussion. Apparently for electronic brainstorming it will be important to note that the intuitive interface is preferred [21]. It is also remarked that for extroverts, the response of other participants in the discussion is important in order to clarify the problem [21]. Participants who have the constructs N - Intuition and E - Extraversion [22] are useful for brainstorming. It is also indicated that it is advisable for students with a combination of ES constructs to be entrusted with case studies based on real facts. And students with a combination of IS constructs are preferable to be entrusted with a critical analysis of real facts and conclusions obtained by other students. In [23], personality types with a combination of IS constructs are characterized as a thoughtful realist, and the practical orientation 
of their thinking is also noted; they are better at solving problems related to real life and based on real facts.

Students with a combination of IN constructs are distinguished by a critical analysis of new ideas, possibilities, and they can also formulate ideas expressed by other participants in the discussion [23]. Personality types with an IN combination are thoughtful innovators, they generate new ideas and penetrate deeply into the essence of the problem, they are most successful at generalizing facts in the form of a concept or theory [23]. The personal type EN shows itself best in new situations; it is an innovator, actionoriented and sees an opportunity to achieve success in challenges [22]. The same characteristic is given in EN [23], it pointed out that this personality type is suitable for joint execution of tasks, including joint generation of ideas during brainstorming.

In [24] give detailed characteristic NT - "Self analysis exercises and structured controversy are more likely to appeal to NTs, who focus on principles and abstractions". In [25] believes that "...NTJ, the type that tends to have the highest grades in engineering and is commonly drawn to engineering research". In [26] supported this characteristic ENTJ "they also have strategic ability, including reasoning, creative problem solving and strategic thinking". In [27] point out "...the student with the ENTJ personality types, it is preferable to choose for the role of a moderator".

The NP personality type likes to participate in brainstorming [21]. In [28] believes that "Sensitive-Thinking (ST) types preferred hard data and logical analysis in reaching decisions" and the "Intuitive-Thinking (IT) types preferred logic and to test several premises before coming to a decision". In [24] describes that "...NT and SJ individuals who tend to be more linear and serial, more structured, more rational and analytical, and more goaloriented in their approach to problem solving." In [24] also describes personality types SP and SJ - "Role-playing and simulations are especially appreciated by SPs and SJs, who generally take a more practical approach to problem solving". For SP advisable it is an iterative process of determining a suitable decision through action. "SJs, care should be taken to proceed in a step-by-step, orderly manner, with ample time for consideration of all details at each step." In [25] add to the characterization of SP and SJ that "...students leave things to the last minute (SP), why some prefer a rigid schedule (SJ), why some are intellectual perfectionists (NT)".

In [26] gives characteristic ENFP as "get involved with the people with whom they work, and are very capable of and willing to reconsider plans when others offer input". For two types ISTJ and ENFP has been described problem-solving characteristics [21]. "In problem solving, ISTJ will want a clear idea of the problem (I) and attack it by looking for the facts (S) and by relying on a logical, impersonal (T), step-by-step approach in reaching conclusions" In [21] and [25] are expressed even more definitely "ISTJ engineering and technology stereotype". An important observation is made that ST and NT is cognitive pairs, they provide the exact opposite of the problem [25]. In [26] notes about ISTJ "meeting deadlines and budgets, ensuring productivity and accountability are strengths of ISTI personality".

ESTJ have a leadership qualities, they characteristics are authority and orientation to results [26]. In [18] supported this think, ESTJ is characteristic as administrator which "possess capability to assimilate large amount detailed information". It is noted that ESTJ is logical and rational; also he knows how to make decisions [18]. It is remarks that the set of INT and EST constructs corresponds to the developer using the heuristic TRIZ method [29]. In particular, ESTJ is suitable "determination of corresponding design principles and reviewing and understanding examples showing appropriate application" and INTP is suitable "abstraction to general TRIZ problem" [29].

\section{The structure of heuristic methods}

The presented overview of heuristic methods does not cover all existing methods. Consideration of only a part of the methods showed that the same method can have different names, such as Affinity Method and K-J method. Either they may differ a little, like Brainwriting and Electronic Brainstorming. The excretion of the characteristic features of methods or their structural units can significantly reduce the number of these methods. From our point of view, the basis of such an analysis can be the excretion of heuristic techniques and procedures from the composition of method. For example, Brainsketching and Method 6-3-5 use the technique of random association. These associations are called through various procedures in the Brainsketching, this is a sketch of another participant, and in the Method 6-3-5 and Brainwriting, these are notes made by another participant. Technique of random associations is used in methods: Brainwriting, Method of focal objects, Method of garlands of accidents and associations, S.C.A.M.P.E.R., Lateral thinking, etc.

The number of heuristic techniques is much less than the number of methods. And within one stage, only a few techniques are used. Let us consider the structure of the methods used in the "Generation phase" stage from the point of view of the applied heuristic techniques. Methods: Brainstorming, Brainwriting, Brainsketching C-Sketch, Gallery, Method 6-3-5, S.C.A.M.P.E.R. be sure to use the collective discussion technique. An important feature of the Brainstorming method is the use of the technique "Pause between the presentation of ideas and their criticism". This technique is also used in methods: Garlands of accidents and associations, Gallery and Lateral thinking. Many methods are based on the use a technique of analogy. These are the following methods: Synectics, Analogy, Empathy and S.C.A.M.P.E.R., which with their questions force the participants to look for similar solutions. In the K-J Method, information is sorted on the basis of analogy, although the use of random associations is also possible. In most methods, the final selection of ideas is done collectively. At the same time, the participants act as experts and their work can be organized on the basis of well-known expert evaluation procedures.

A powerful analysis tool is a heuristic technique «Use of matrix". In different versions, this technique is used by methods: Morphological analysis, DSM matrix, Interaction matrix, Matrix diagram, AIDA. First of all, the methods are selected that are suitable for use in the "Generation phase" stage, are widely known and contain, if possible, several heuristic techniques. These methods are summarized in the table to analyze their structures (Table 1). 
Table 1: Structure of heuristic methods

\begin{tabular}{|c|l|l|l|l|l|l|}
\hline $\begin{array}{r}\text { Heuristic } \\
\text { techniques } \\
\text { methods }\end{array}$ & $\begin{array}{c}\text { Collective } \\
\text { discussion }\end{array}$ & $\begin{array}{c}\text { Pause between the } \\
\text { presentation of ideas } \\
\text { and their criticism }\end{array}$ & $\begin{array}{c}\text { Random } \\
\text { associations }\end{array}$ & Analogy & $\begin{array}{c}\text { Expert } \\
\text { evaluation }\end{array}$ & $\begin{array}{c}\text { Using a } \\
\text { matrix }\end{array}$ \\
\hline Brainstorming & & & & & & \\
\hline Brainwriting & & & & & & \\
\hline Method 6-3-5 & & & & & & \\
\hline S.C.A.M.P.E.R. & & & & & & \\
\hline Brainsketching & & & & & & \\
\hline Gallery & & & & & & \\
\hline Storyboarding & & & & & \\
\hline C-Sketch & & & & & & \\
\hline Lateral thinking & & & & & & \\
\hline Method of focal objects & & & & & & \\
\hline $\begin{array}{c}\text { Method of garlands of } \\
\text { accidents and } \\
\text { associations }\end{array}$ & & & & & & \\
\hline Synectics & & & & & & \\
\hline Affinity Method & & & & & & \\
\hline Lateral thinking & & & & & & \\
\hline Morphological Matrix & & & & & & \\
\hline DSM matrix & & & & & & \\
\hline Interaction matrix & & & & & & \\
\hline Matrix diagram & & & & & & \\
\hline AIDA & & & & & & \\
\hline
\end{tabular}

To develop a generalized heuristic method aimed at solving problems within the framework of the stage, we formulate a theorem.

Theorem: for each stage of the creative process one generalized heuristic method can be applied replacing all methods used for this stage if it includes all heuristic techniques used within this stage.

The generalized method for "Generation phase" stage includes the following heuristic techniques: collective discussion, pause between the presentation of ideas and their criticism, random associations, analogy, expert evaluation, use of matrix. The use of all these techniques in one method is mandatory. The order of using the techniques and the procedures applied in the method may be different depending on the branch of knowledge and the problems being solved. For example, the "Collective discussion" technique can be applied twice. Then one gets a variation of the brainstorming method - double brainstorming. The Brainsketching and Gallery methods use the "Random association" technique. These methods use different procedures to enhance creativity. In the Brainsketching method, participants can flip the image upside down, and in the Gallery method, they can observe the sketching process. The methods of the DSM matrix and the Matrix Diagram use the same heuristics "Using a matrix" and different procedures. In the DSM matrix, the procedure is to establish the presence or absence of an interaction between elements, and in the Matrix Diagram, the procedure is to assess the conformity of an element to certain requirements.
Let's point out the procedures typical for the methods used in teaching. These are procedures: selection of a moderator, selection of participants in a discussion group, selection of members of a group of critics, selection of members of the group of expert evaluation. Also, the procedures are - defining the rules of discussion and the method of forming an expert evaluation. Important procedures are the choice of the physical meaning of the columns and rows of the matrix and the rules for evaluating combinations of row and column elements.

Based on the analysis of heuristic techniques and procedures, it is possible to form an algorithm of the generalized method. This method must be suitable for use in education, also for design. That is, it should be a method of increasing creativity and at the same time a method suitable for use in the design process. The latter is important for students engineering specialties. To combine such an algorithm and data on the predisposition of students of different personality types to the use of heuristic techniques and procedures in the generalized method, it is important to establish what personality types are typical for students studying in STEM specialties.

\section{Typical personality types of students STEM specialties}

It is known that there are more introverts than extroverts among students studying STEM specialties. Thus, among students studying agricultural engineering, $83 \%$ have construct I, and only $17 \%$ have construct $\mathrm{E}$ [21]. In the ratio of the $\mathrm{S}$ and $\mathrm{N}$ constructs, there is no unambiguity: the construct $\mathrm{S}$ prevails in mechanical engineering $(64 \mathrm{~S} \%, 36 \% \mathrm{~N})$, while for aerospace engineering the 
construct $\mathrm{N}$ prevails. The most obvious is the predominance of the construct $\mathrm{T}$ over $\mathrm{F}$ and $\mathrm{J}$ over $\mathrm{P}$ [21].

It is claimed those students with the following personality types: ENTJ, INTJ, INTP, ESTJ, ISTJ, ESTP and ISTP, have the ability to work in engineering positions [30]. The personality type of ISTJ is especially highlighted, which is predominant among students of engineering specialties and makes up $26 \%$ of their total number [26]. Based on research carried out in [32], it is also stated that the personality type ISTJ occurs most often, the second most common is one of the following personality types ESTJ, INTP and INTJ. Testing of students according to typology (MBTI) was conducted in eight universities [21]. The percentage of students of a certain personality type is established, it is indicated in brackets. The study showed that personality types: ISTJ $(16.46 \%)$, ESTJ (12.75\%), ENTJ (9.43\%), INTJ (9.43\%), INTP (8.46\%) and ENTP (7.43\%), most often found among students studying STEM disciplines [21]. In total, personality types ISTJ, ESTJ, INTJ and INTP make up more than $50 \%$ of the students who passed the test, and personal types ESFP, ESFJ and ENFJ are less common among students of STEM specialties [31]. The most detailed study of the representation of certain personality types among students of STEM specialties is presented in [10]. First of all, we note that the most widespread personality type is ISTJ $(16.9 \%)$, and the second place is occupied by INTJ $(12.3 \%)$, which corresponds to the research data [32]; not much less than INTJ is represented by ENTP (11.8\%); for other personality types the following data were received: ESTJ (9.2\%), ISTP (7.7\%) and INTP (7.2\%) [10]. Thus, only four personality types out of sixteen ISTJs, INTJs, ENTPs and ESTJs account for $50.2 \%$ of the total number of students. The data of another survey [33] also revealed the prevalence of ISTJ $(18.1 \%)$, and the second place in prevalence was taken by ESTJ $(10.3 \%)$, as in [21]. Other personality types ranked in the following sequence: INTP $(9.4 \%)$, INTJ ( $8.5 \%)$, ISTP $(8.2 \%)$. It is noted that these five personality types make up more than half of the students [33]. It is noted that these five personality types account for more than half of the students [33]. Similar data were obtained in [32] the following personality types are prevalent among students of STEM specialties: ISTJ, INTP, ESTJ, INTJ, ENTP, ENTJ and ISTP. Obviously, in further research, it is necessary to obtain data on the possibility of participation of precisely these personality types at different stages of heuristic search. According to the sources discussed above, the following personal types are typical for students STEM professions: ISTJ, ESTJ, ENTJ, INTJ, INTP и ENTP. It is also found that four personal types can account for more than half of the total. Three of them are ISTJ, ESTJ, INTJ, as the fourth, both INTP and ENTP are mentioned. For developing a heuristic method that uses personal types in a particular way, these facts are very important.

Therefore, a study of the distribution of personal types among students speciality "Mathematics and Physics" at the South Ukrainian National Pedagogical University named after K. D. Ushynsky (SUNPU) was carried out. The same test was performed for students speciality "Dynamics and durability of machines" Odessa National Polytechnic University (ONPU) in 2014-2016 years. In each semester, two academic groups numbering from 15 to 20 students were tested. The test was conducted twice, at the beginning and at the end of the semester. The coincidence of test results was observed in the range of $76-81 \%$, which is within acceptable limits (75-90\%) [34].

www.astesj.com
There were no students who refused to participate in the survey. But among the students of ONPU there were also those who did not take it seriously. Their questionnaires were not taken into account. Students of SUNPU treated the survey more scrupulously when the purpose of the survey was clarified - it was the selection of students into project team. They showed a great interest in testing, as it is directly related to their prospective teaching profession.

The distribution of personality types was calculated twice, first for all students, then separately, only for men majoring in "Dynamics and Durability of Machines" (ONPU), where the proportion of men is $87 \%$, and only for women majoring in "Mathematics and Physics" "(SUNPU), where the share of women is $72 \%$. The average value of data of the first and retesting were calculated. In that case, if the student equally corresponded to two personality types, for example, the constructs $\mathrm{T}$ and $\mathrm{P}$ scored the same number of points, then such people were taken into account twice, in each of the personality types. There were students who did not care about the test and gave mutually exclusive answers; such questionnaires were not taken into account in further calculations. The test results are summarised at Table 2.

Table 2: Personality types of ONPU and SUNPU students

\begin{tabular}{|c|cc|}
\hline $\begin{array}{c}\text { Personality } \\
\text { types }\end{array}$ & $\begin{array}{c}\text { Students } \\
\text { ONPU (\%) }\end{array}$ & $\begin{array}{l}\text { Students } \\
\text { SUNPU (\%) }\end{array}$ \\
\cline { 1 - 2 } ISTJ & 22,1 & 18,1 \\
\cline { 1 - 1 } INTP & 11,9 & 15,5 \\
\cline { 1 - 1 } ESTJ & 11,7 & 14,7 \\
\cline { 1 - 1 } INTJ & 11,6 & 10,4 \\
\cline { 1 - 1 } ENTP & 7,8 & 8,4 \\
\cline { 1 - 1 } ENTJ & 5,3 & 4,4 \\
\cline { 1 - 1 } ISTP & 4,2 & 3,3 \\
\cline { 1 - 1 } ENFP & 2,0 & 2,5 \\
\hline
\end{tabular}

ISTJ personality types are more common in ONPU, but INTP and ESTJ personality types are more common in SUNPU, as shown in Figure 1.

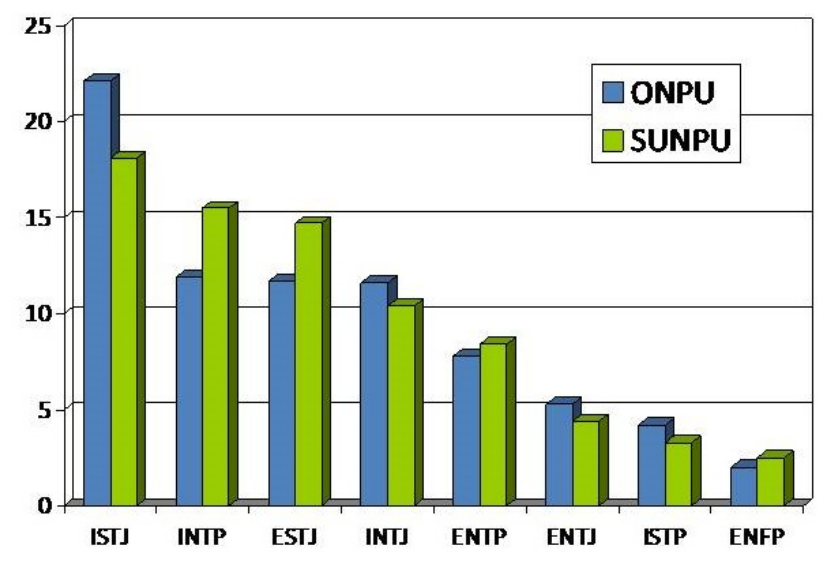

Figure 1: Percentage distribution of personality types

The relationship between personal types can be most clearly represented in the form of a pie chart in which the axes correspond to certain cognitive functions. The abscissa corresponds to the cognitive functions $\mathrm{N}$ and $\mathrm{S}$. The construct $\mathrm{N}$ is located on the left, and the constructs $\mathrm{S}$ is located on the right along the abscissa. The 
ordinate corresponds to the cognitive functions $\mathrm{T}$ and $\mathrm{F}$. The construct $\mathrm{T}$ is placed below the abscissa, and the construct $\mathrm{F}$ above the abscissa is along the ordinate. The personality types within the inner circle are introverted; personality types outside the inner circle are extroverts. For each personality type, the average value of students of this type in ONPU and SUNPU was calculated: ISTJ (20.1), INTP (13.7), ESTJ (13.2). INTJ (11.0), ENTP (8.1), ENTJ (4.9), ISTP (3.8) and ENFP (2.3) (Figure 2).

The obtained average values of personality types were plotted on this pie chart. The size of the circle corresponds to the percentage of this personality type among all students.

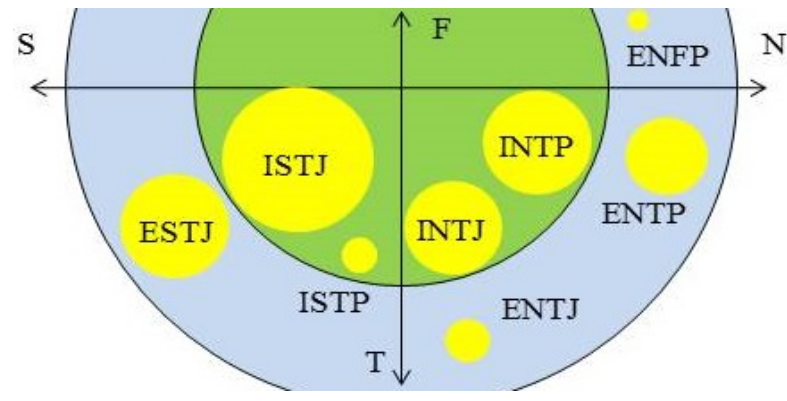

Figure 2: Distribution of personality types relative to the pie chart depicting cognitive functions

The small number of students surveyed does not allow making any conclusions about gender differences. The repeated survey for women in ONPU and men in SUNPU gave an error higher than acceptable. We cannot fully rely on the absolute value of a particular personality type. Because when surveying 50 students, one incorrectly completed questionnaire gives an error of $2 \%$. The data of students at six technical universities in the United States and Canada shows that only one university has the represented ENFP type among students [32]. For this reason, we excluded the ENFP personality type from further consideration because the data obtained correspond to one representative from two academic groups.

We obtained the following order of personal types by their prevalence: ISTJ, INTP, ESTJ, INTJ, ENTP, ENTJ and ISTP. The first is ISTJ, as in the sources [21, 26, 31]. The first six personal types are the same as in [21], but their order is different. The resulting distribution of personal types is close to [33]. In our study, the INTP (13.7\%) is in second place and the ESTJ (13.2\%) is in third place. the difference between them is within the margin of error. In [33] type personal ESTJ $(10,3 \%)$ at the second location, and INTP $(9,4 \%)$ for the third, but the difference between them is small. In [31], also the personal type ESTJ (10.9\%) is in second place, and INTP $(9.9 \%)$ is in third place and the difference between them is about $1 \%$ as in the article [33]. The first four personal types in our study make up 58\%, which is also more than half of the total number as in sources [31,33]. According to our data, more than $75 \%$ of students STEM specialties belong to the following personality types: ISTJ, INTP, ESTJ, INTJ, ENTP, ENTJ and ISTP. The personality types in the table are arranged in descending order, in accordance with the number of representatives of this type (Table). Refer that the most common personality types include types containing the construct $\mathrm{T}$. These personality types have a predisposition to STEM professions. The only personality type between of those which contain a construct $\mathrm{T}$ and which is ranked among the common - this ESTP. This type is also not mentioned among those common in papers $[21,33]$. This is due to the fact that students of this type neglect the details and do not finish what they started. Equally represented are constructs Sensing (S) and Intuition $(\mathrm{N})$.

\section{The generalized method}

The generalized method contains a heuristic technique using $a$ matrix. Rows and columns of a matrix can be functions, units, a number of products with similar functions, design techniques, and so on. These recommendations are intended to solve engineering problems. The range of tasks solved by the technique using a matrix is much wider. It is proposed that the purpose of the technique collective discussion is to find ideas about the contents of the columns and rows of the matrix. After the first heuristic technique, there is a pause between the presentation of ideas and their criticism. In further, the criticism of the ideas put forward and their evaluation are providing. That is, the technique of expert evaluation is used. The semantic content of rows and columns is searched for by the technique of analogy and random associations. For example, for rows, the search is carried out by analogy, and for columns using random associations. If the search did not give satisfactory results, it is repeated, but the technique of random associations is already used for rows, and analogy for columns. The results are again collectively discussed and undergo expert evaluation. Based on the results of the heuristic analysis, the technique of using a matrix is applied - in this way the matrix is formed. Based on the results of the heuristic analysis, a technique using a matrix is applied - therefore the matrix is analyzed. Each cell of the matrix corresponds to a possible variant of a problem solution. These options are subject to collective discussion again. Then, expert evaluation and documenting of the results are performed.

The heuristic techniques and procedures described above require performers with a certain personal type. An effective collective discussion technique will not work if all participants are introverts. Extroverts with artistic abilities should not be entrusted with documenting matrix analysis results. The complete development of the method requires specifying the personality type in the performers for each of the techniques and procedures.

The ENTJ personality type has leadership qualities, strategic vision, logic, and therefore can be a moderator of the discussion. ESTJ - has organizational skills and the ability to keep track details of discussion. A student with the ENTJ personality type is selected as the moderator. If there was no such student in the group, then the student with the ESTJ personality type plays the role of the moderator. The moderator participates in the collective discussion.

Collective discussion is an extroverted process. Intuition is also important for generating ideas. In the process of collective discussion, there is a search for patterns, the construction of schemes and models, and a search for the relationship between known facts. It is important that the participants discard wellknown dogmas, abandon stereotypes. Extroverted personality types (construct E) and intuitive personality types (construct $\mathrm{N}$ ) take part in the collective discussion. Preference is given to personality types ENTJ, ENTP.

The logic is inherent by personality $\mathrm{T}$. The personality NT is inventive, looking for regularities. The personality types INTJ and 
INTP can generate ideas, however, they are introverts and the collective discussion is a problem for them. The personality type INTP is actively generating ideas; he has logic (T) and the ability to evaluate the logic of ideas expressed by other members of the team. The personality type INTJ is a researcher and has good intuition, but due to communication problems with others, he is not always possible to clearly express their ideas. The personality type INTP can be tactless and arrogant, which hinder a spontaneous expression of ideas. Based on the foregoing, the types of personality INTP perform the function of criticism.

The understanding an importance of the knowledge is inherent of a personality type TE. This quality, as well as extraversion, allows the personality type ESTJ to participate in the discussion, although ESTJ can consider the brainstorming ineffective. Based on the analysis of the above, students with personality types ENTJ, ENTP and INTJ are necessarily selected for the discussion group.

For the critical analysis of the proposed ideas, the following qualities are important: distrust, searching for flaws in everything, attention to detail, logicality. The critic is, without a doubt, an introvert type. The personality types ISTP, ISTJ, INTP, and INTJ are logical introverts. Critical analysis is the strong point of IS and IN. Sensitive-Thinking personality types ISTP and ISTJ prefer to focus on practical issues. These personality types prefer to carefully compare several options before making a final decision. In doing so, they rely on facts and logical analysis. Personality types INTP and INTJ are perfectionists that important for critics. Based on the foregoing, the students with the types of personality ISTP, INTP and ISTJ can function as critics.

Expert evaluation and critical analysis require similar personality types. Personality types ST and NT are a cognitive pair. For example, these are the ISTJ and INTJ personality types. They provide a different vision of the problem, this is important for the evaluation. The final decision on the meaning of rows and columns is made primarily by "experts" in the first place; these are representatives of the personality types ISTJ, and also ENTJ, ESTJ. The inclusion of personality types ENTJ and ESTJ in the number of experts is due to the fact that they led a collective discussion. The moderator can choose the final variant basing not only on his own opinion, but also on the opinion of other members of the team whose personal qualities he knows and appreciates. Therefore students with personality types ISTJ, INTJ, ESTJ and ENTJ can participate in the expert evaluation.

Note that the ENTJ and ENTP personality types have a good imagination and are useful when using the technique of random associations, and the INTJ personality type is endowed with perfect intuition. The ISTJ personality type is an ideal candidate for processing the results of the discussion and organizing them in the form of a morphological matrix of proposed solutions. The personal INTP type can do the job as well. It is also advisable to entrust the documentation of the final results obtained using the generalized method to the personal types of ISTJ and INTP.

If the proposed heuristic method does not work, a Feedback Group can be created. It is advisable to include representatives of each of the 16 personality types in this group, if students with such personal types are available. A group of students who performed a certain heuristic technique told the Feedback Group about their experience of participation, as well as problems that arose. After the discussion, all or part of the heuristic techniques are repeated.

Consider the algorithm of the generalized heuristic method, taking into account the heuristic techniques and the content of the considered procedures (Figure 3).

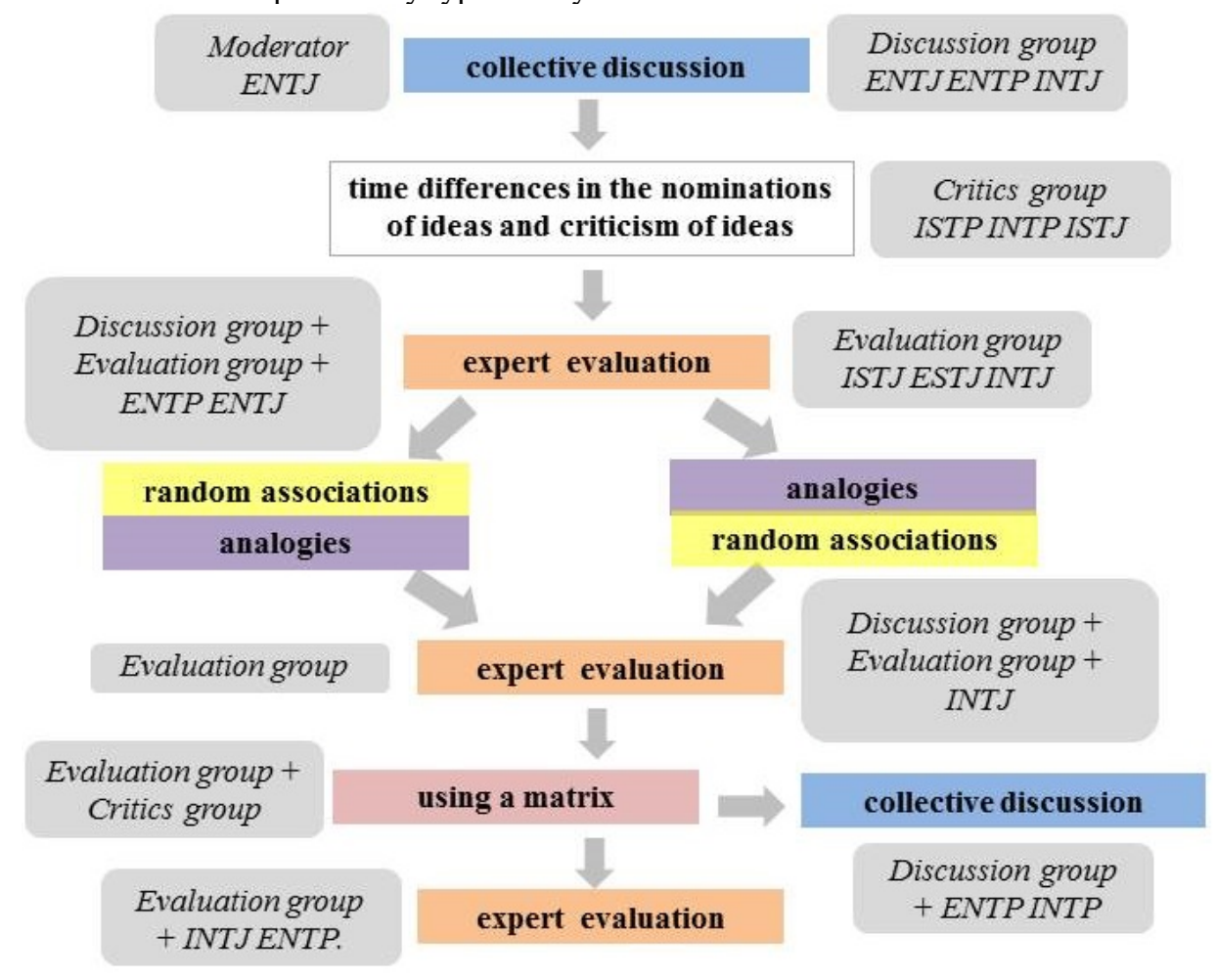

Figure 3: The algorithm of generalized method 
The first procedure is procedure for selecting a moderator. A student with personality types ENTJ is selected as moderator for the collective discussion group. If there are two representatives of this type, then the student with higher academic achievements is chosen as the moderator. If there are no representatives of this personality type, then ESTJ is chosen as the moderator. The project team should be divided into several parts: discussion group, critics group, evaluation group. The one student can be by participant of few groups.

The second procedure is procedure for selecting participants for the discussion group; these are personality types ENTJ, ENTP and INTJ.

The third procedure for selecting the participants of critics group is as follows - students with the ISTP, INTP and ISTJ personality types are selected as critics group.

The fourth procedure for selecting the participants of expert evaluation group: personality types ISTJ, ESTJ, and INTJ.

This is followed by the first heuristic technique - collective discussion. The collective discussion group consisted of five to ten people. Personality type ESTJ participated in discussions and idea generation provided he is a moderator. This personality type joins the discussion group also in the case of its small size.

The second heuristic technique is a pause between the presentation of ideas and their criticism. After a pause, the group of critics set to work. A group of critics is consisted of three to five people. The composition of the group can be increased so that all members of the project team can participate in the work. In small project teams with a lack of "critics", the ISTJ is served as a critic; they usually predominate numerically over representatives of other personality types. In this case, the INTP is joined the group of discussion and generation of ideas. Several members of the collective discussion group join the group of critics. First of all, this is the personal type of INTJ, Thus, a critical analysis is carried out both by the project team members who participated in the discussion and those who did not participate in the discussion; they represent a different point of view on the problem.

The third heuristic technique is expert evaluation. The group of experts should not be large. Not all participants with personality types ISTJ, ESTJ, ENTJ should be members of this group. This group primarily includes students with high academic achievement. Moderator is always included in this group.

The fifth procedure is the selection of groups for heuristic techniques of analogy and random associations. These groups are mainly formed from members of the discussion group and expert evaluation group. But members of the critics' group also participated, so almost all members of the project team participated in the two groups. Personality types of ENTJ and ENTP should be included in the group of random associations, and the group of analogy - personal type INTJ.

The fourth heuristic technique - analogy and the fifth heuristic technique - random associations were used in parallel and repeated if necessary.

The sixth heuristic technique is expert evaluation. The group can be updated. Other members of the personality types ISTJ, ESTJ and INTJ may be included in this group.
The seventh heuristic technique is the using a matrix. The matrix is formed based on the results of applying the previous heuristic techniques. For the technical work on the formation of the matrix, personality types ISTJ and INTP are involved. An evaluation group and a critics group analyze each cell of the matrix. Decisions that do not make sense and are not feasible under the given conditions are discarded. Promising solutions are brought up for discussion.

The eighth heuristic technique is a collective discussion. The discussion group can be expanded. You can add personality types with good logic - ENTP and INTP. Note that INTP was involved in the matrix developing.

The ninth heuristic technique is an expert evaluation. This is final heuristic technique and final evaluation. In this case, the evaluation group should be expanded to include representatives of discussion group: INTJ and ENTP. As a result of considering the options detailed in the process of collective discussion, one best is chosen.

Final procedure is a documenting the results. For this the personality types ISTJ and INTP. To do this, personality types INTJ and INTJ are involved.

\section{Conclusion}

The analysis of heuristic methods based on their structure is carried out. The heuristic methods which belong to the generation phase were considered. As part of the methods highlighted heuristic techniques and procedures. It is shown that two dozen methods belonging to the generation phase use only five heuristic methods. Many methods use the same heuristic technique and differ only in procedure. The theorem was formulated: for each stage of the creative process one generalized heuristic method can be applied replacing all methods used for this stage if it includes all heuristic techniques used within this stage.

The study of the personality type's distribution of students STEM specialties was carried out at the South Ukrainian National Pedagogical University named after K. D. Ushynsky (SUNPU) and Odessa National Polytechnic University (ONPU). The main part of students belongs to seven personality types. Data for SUNPU students on the percentage of each type to the total number of students: ISTJ (18.1), INTP (15.5), ESTJ (14.7). INTJ (10.4), ENTP (8.4), ENTJ (4.4), ISTP (3.3) and ENFP (2.5). Data for ONPU students on the percentage of each type to the total number of students: ISTJ (22.1), INTP (11.9), ESTJ (11.7). INTJ (11.6), ENTP (7.8), ENTJ (5.3), ISTP (4.2) and ENFP (2.0). The generalized method was developed. This method focused to teaching students STEM specialties. Method based on five heuristic techniques collective discussion, pause between the presentation of ideas and their criticism, random associations, analogy, expert evaluation, using a matrix. The heuristic process is considered from the point of view of the personality types of the participants. Personal types are considered in terms of how they are suitable for performing the indicated heuristic techniques. The recommendation about performers: moderator, discussion group, critics group, evaluation group, for each heuristic technique was developed.

\section{Conflict of Interest}

The authors declare no conflict of interest. 


\section{Acknowledgments}

This work has been accomplished with financial support by the Grant No BG05M2OP001-1.002-0011 "MIRACle (Mechatronics, Innovation, Robotics, Automation, Clean technologies)", financed by the Science and Education for Smart Growth Operational Program (2014-2020) and co-financed by the European Union through the European structural and Investment funds.

\section{References}

[1] V. Ivanov, L. Dimitrov, S. Ivanova, O. Olefir, "Creativity enhancement method for STEM education," in 2019 IEEE International Conference on High Technology for Sustainable Development (HiTech), 1-5, 2019, doi: 10.1109/HiTech48507.2019.9128255.

[2] J.S. Linsey, M.G.Green, J.T. Murphy, K. L. Wood, A.B. Markman, "Collaborating To Success: An Experimental Study of Group Idea Generation Techniques," in International Design Engineering Technical Conferences and Computers and Information in Engineering, 4742, 277-290, 2005, https://doi.org/10.1115/DETC2005-85351.

[3] R. Van der Lugt, "Brainsketching and how it differs from brainstorming," Creativity and innovation management, 11(1), 43-54, 2002, https://doi.org/10.1111/1467-8691.00235.

[4] F.L. McKoy, N. Vargas-Hernández, J.D. Summers, J.J. "Shah, Influence of design representation on effectiveness of idea generation," in Proceedings of the ASME design engineering technical conference, 4, 39-48, 2001.

[5] M. Fargnoli, E. Rovida, R.Troisi, "The morphological matrix: Tool for the development of innovative design solutions," in 2006 International Conference on Axiomatic design (ICAD), 1-7, 2006.

[6] Y.J. Park, C. Kim, J. Yoon, "Creativity and design method in idea generation: the comparison between intuitive approach vs structured approach," in 2019 IASDR Conference, 1-12, 2019.

[7] V. Chulvi, M.C. González-Cruz, E. Mulet, J. Aguilar-Zambrano, "Influence of the type of idea-generation method on the creativity of solutions," Research in Engineering Design, 24(1), 33-41, 2013, https://doi.org/10.1007/s00163-012-0134-0.

[8] A. Weas, M. Campbell, "Rediscovering the analysis of interconnected decision areas," AI EDAM, 18(3), 227-243, 2004, https://doi.org/10.1017/S0890060404040168.

[9] W. Beitz, G. Pahl, K. Grote, "Engineering design: a systematic approach," MRS Bulletin, 21(8), 71-71, 1996, doi:10.1557/S0883769400035776.

[10] T.J. Howard, S.J. Culley, E. Dekoninck, "Describing the creative design process by the integration of engineering design and cognitive psychology literature," Design studies, 29(2), 160-180, 2008, https://doi.org/10.1016/j.destud.2008.01.001.

[11] S. Buzuku, A. Kraslawski, "Use of Design structure matrix for analysis of critical barriers in implementing eco-design initiatives in the pulp and paper industry," Procedia Manufacturing, 11, 742-750, 2017, https://doi.org/10.1016/j.promfg.2017.07.175.

[12] V. Ivanov, G. Urum, S. Ivanova, G. Naleva, "Analysis of matrix and graph models of transmissions for optimization their design," Eastern - European Journal of Enterprise Technologies, 4.1(88), 11-17 2017, https://doi.org/10.15587/1729-4061.2018.131101.

[13] C. Pokhrel, C. Cruz, Y. Ramirez, A. Kraslawski, "Adaptation of TRIZ contradiction matrix for solving problems in process engineering," Chemical engineering research and design, 103, 3-10, 2015, https://doi.org/10.1016/j.cherd.2015.10.012.

[14] Vezzetti, F. Marcolin, A.L. Guerra, "QFD 3D: a new C-shaped matrix diagram quality approach," International Journal of Quality \& Reliability Management, 33 (2), 178-196, 2016, https://doi.org/10.1108/IJQRM-072013-0112.

[15] D. Lukianov, K. Bespanskaya-Paulenko, V. Gogunskii, O. Kolesnikov, A. Moskaliuk and K. Dmitrenko "Development of the markov model of a project as a system of role communications in a team," Eastern-European Journal of Enterprise Technologies, 3(3), 21-28, 2017, https://doi.org/doi:10.15587/1729-4061.2017.103231.

[16] M. Farhangian, M. Purvis, M. Purvis, T.B.R. Savarimuthu, "Agent-based modeling of resource allocation in software projects based on personality and skill," Advances in Social Computing and Multiagent Systems. MFSC 2015. Communications in Computer and Information Science, 541, 130-146, 2015, https://doi.org/10.1007/978-3-319-24804-2_9.

[17] N. Lehmann-Willenbrock, S.J. Beck, S, Kauffeld, "Emergent team roles in organizational meetings: Identifying communication patterns via cluster analysis," Communication Studies, 67(1), 37-57, 2016, https://doi.org/10.1080/10510974.2015.1074087.

[18] J. Jankowski, The 16 Personality Types in a Nutshell, LOGOS MEDIA, 2016.

[19] M.H. McCaulley, "The Myers-Briggs type indicator: A Jungian model for problem solving." New Directions for Teaching and Learning, 1987(30), 37 53, 1987, https://doi.org/10.1002/tl.37219873005.

[20] S. Rushton, J. Morgan, M. Richard, "Teacher's Myers-Briggs personality profiles: Identifying effective teacher personality traits," Teaching and Teacher Education, 23(4), 432-441, 2007, https://doi.org/10.1016/j.tate.2006.12.011.

[21] M.H. McCaulley, "Myers-Briggs Type Indicator: A bridge between counseling and consulting," Consulting Psychology Journal: Practice and Research, 52(2), 117-132, 2000, https://doi.org/10.1037/10614087.52.2.117.

[22] D. Keirsey, M.M. Bates, Please understand me: Character \& temperament types, Del Mar, CA: Prometheus Nemesis Book Company, 1984.

[23] C. Soles, L. Moller, "Myers Briggs type preferences in distance learning education," International Journal of Educational Technology, 2(2), 2001.

[24] W. Huitt, "Problem solving and decision making: Consideration of individual differences using the Myers-Briggs Type Indicator," Journal of Psychological Type, 24(1), 33-44. 1992.

[25] C.F. Yokomoto, R. Ware, "Applications of the Myers-Briggs Type Indicator in engineering and technology education - part II," Indiana University Purdue University Indianapolis, 2230, 4.88.1- 4.88.10, 1999.

[26] V.R. Montequín, J.V. Fernández, J.V. Balsera, A.G. Nieto, "Using MBTI for the success assessment of engineering teams in project-based learning," International journal of technology and design education, 23(4), 1127-1146, 2013, https://doi.org/10.1007/s10798-012-9229-1.

[27] S. Ivanova, L. Dimitrov, V. Ivanov and G. Naleva, "An Experiment on the Joint use of the Heuristic and Project Methods at the University," in 2019 IEEE International Conference on High Technology for Sustainable Development (HiTech), 1-5, doi: 10.1109/HiTech48507.2019.9128248.

[28] J.B. Murray, "Review of research on the Myers-Briggs type indicator," Perceptual and Motor skills, 70(3_suppl), 1187-1202, 1990, https://doi.org/10.2466/pms.1990.70.3c.1187.

[29] M. Ogot, G. Okudan, "A student centered approach to improving course quality using quality function deployment," The International journal of engineering education, 23(5), 916-928, 2007.

[30] P.D. Tieger, B. Barron, K. Tieger, Do what you are: Discover the perfect career for you through the secrets of personality type. Little, Brown Spark, 2014.

[31] A.J. Williamson, "Suiting library instruction to the Myers-Briggs personality types and Holland Vocational personality types of engineering students," Issues in Science and Technology Librarianship, 37(2), 2003. https://doi.org/10.5062/F4154F1G.

[32] L.F. Capretz, "Is there an engineering type?" World Transactions on Engineering and Technology Education, 1(2), 169-172, 2002.

[33] A. Thomas, M.R. Benne, M.J. Marr, E.W. Thomas, R.M. Hume, "The evidence remains stable: The MBTI predicts attraction and attrition in an engineering program," Journal of Psychological Type, 55, 35-42, 2000.

[34] R.M. Capraro, M.M. Capraro, "Myers-Briggs Type Indicator score reliability across studies: A metaanalytic reliability generalization study," Educational and Psychological Measurement, 62(4), 590-602, 2002. 